\title{
A NOTE ON THE DIVIDENDS-PENALTY IDENTITY AND THE OPTIMAL DIVIDEND BARRIER
}

BY

\author{
Hans U. Gerber, X. Sheldon Lin and Hailiang Yang
}

\begin{abstract}
For a general class of risk models, the dividends-penalty identity is derived by probabilistic reasoning. This identity is the key for understanding and determining the optimal dividend barrier, which maximizes the difference between the expected present value of all dividends until ruin and the expected discounted value of a penalty at ruin (which is typically a function of the deficit at ruin). As an illustration, the optimal barrier is calculated in two classical models, for different penalty functions and a variety of parameter values.
\end{abstract}

\section{KEYWORDS}

General class of risk models, dividends-penalty identity, penalty at ruin, optimal dividend barrier, Brownian motion, compound Poisson process.

\section{INTRODUCTION}

In recent years, there has been considerable renewed interest in insurance risk models with dividend strategies. Most studies focus on a specific model, and their approaches are more or less model-specific. The purpose of this note is to consider a general class of risk models and to explain basic concepts by probabilistic interpretations. Thus heuristic reasoning will be preferred to mathematical formalism (and we count on the tolerance of the mathematical purists). In particular, the dividends-penalty identity will be derived with a minimal mathematical effort.

Let $U(t)$ be the surplus of a company at time $t$ and $U(0)=u$ the initial surplus. Dividends are paid to the shareholders of the company according to some strategy. In particular, we consider barrier strategies. The parameter of such a barrier strategy is denoted by $b$. Then, whenever the surplus is about to surpass the level $b$, the potential excess above $b$ is immediately paid out as dividends. As a consequence, the original surplus process is replaced by the modified surplus process, which takes into account the dividend payments. Ruin occurs when the modified surplus becomes negative for the first time. 
Surplus processes with barrier dividend strategies were considered in Bühlmann (1970), Gerber and Shiu (1998), Lin et al. (2003), Li and Garrido (2004), Albrecher et al. (2005), Frostig (2005), Yuen et al. (2005), to name a few.

The basic assumption in this note is that the surplus process $\{U(t)\}$ is a stationary Markov process and is skip-free upwards. Thus, the sample paths may have jumps downwards, but not upwards. This has the following implication: let $u_{1}<u_{2}<u_{3}$ be three surplus levels; if the surplus passes from $u_{1}$ to $u_{3}$, it must pass through $u_{2}$. As in the classical risk theory, the surplus process could be a shifted compound Poisson process, where the surplus at time $t$ is

$$
U(t)=u+c t-S(t),
$$

where $c$ is the premium rate, and the aggregate claims process $\{S(t)\}$ is compound Poisson with Poisson parameter $\lambda$ and claim amount distribution $P(x)$. However, the model of this paper is much more general. Under the stationary Markovian assumption, we allow the premium rate, the Poisson parameter, and the claim amount distribution to depend on the current value of the surplus. Furthermore, a diffusion component could be added to the model, and a stochastic investment return on the surplus could also be part of the model.

This note is organized as follows. Sections 2 and 3 provide a succinct derivation of the dividends-penalty identity in its general form. As a consequence, the optimal dividend barrier is well defined; this is discussed in Sections 4 and 5. In Sections 6 and 7, the optimal dividend barrier is calculated for two classical models and under different assumptions about the parameter values and the penalty function.

\section{THE BARRIER STRATEGY}

We introduce the function $C\left(u_{1}, u_{2}\right)$, which is defined for surplus levels $u_{1} \leq u_{2}$. In the following, this function will serve as a basic building block. For initial surplus $u_{1}$, we define $C\left(u_{1}, u_{2}\right)$ as the expected present value of a contingent payment of 1 due at the time when the surplus reaches the level $u_{2}>u_{1}$, provided that ruin has not occurred in the meantime; we set $C(u, u)=1$. Hereafter, the words "present value" and "discounted value" of one or several payments refer to the same constant force of interest $\delta>0$, which will not be mentioned explicitly. Note that the function $C\left(u_{1}, u_{2}\right)$ resembles the net single premium of a pure endowment policy in life contingencies. By "general reasoning" (with a tip of the hat to the strong Markov property) it is seen that

$$
C\left(u_{1}, u_{3}\right)=C\left(u_{1}, u_{2}\right) C\left(u_{2}, u_{3}\right), \quad u_{1} \leq u_{2} \leq u_{3} .
$$

This shows that there is a positive increasing function $h(x)$ such that

$$
C\left(u_{1}, u_{2}\right)=\frac{h\left(u_{1}\right)}{h\left(u_{2}\right)} \quad \text { for } u_{1} \leq u_{2} .
$$


The function $h(x)$ is unique apart from a constant factor. We may choose any $u_{0}$ and set $h\left(u_{0}\right)=1$. Then

$$
h(u)= \begin{cases}C\left(u, u_{0}\right) & \text { if } u<u_{0} \\ \frac{1}{C\left(u_{0}, u\right)} & \text { if } u>u_{0} .\end{cases}
$$

It is now assumed that dividends are paid to the shareholders according to the barrier strategy with parameter $b>0$. Let $V(u ; b), u \leq b$, denote the expected present value of all dividends until ruin, where $u$ is the initial surplus. By "general reasoning" we see that

$$
V(u ; b)=C(u, b) V(b ; b), \quad u \leq b .
$$

Moreover, the (left) derivative of $V(u ; b)$ at $u=b$ is

$$
V^{\prime}(b-; b)=1 \text {. }
$$

Condition (5) is best explained by the following heuristic argument. Compare two situations, situation 1 where the initial surplus is at the barrier $b$, and situation 2 where the initial surplus is $b-\varepsilon(\varepsilon>0$ and "small"). It is quasi certain that the surplus in situation 2 reaches the barrier "shortly" (and before ruin). Under situation 1, a total dividend of $\varepsilon$ will have been paid by then. After this time, the surplus processes, and in particular the dividend payments, are the same in situations 1 and 2. It follows that the difference $V(b ; b)-V(b-\varepsilon ; b)$ is essentially $\varepsilon$, which explains (5).

From (3)-(5), it follows that

$$
V(u ; b)=\frac{h(u)}{h^{\prime}(b)}, \quad 0 \leq u \leq b .
$$

For the classical compound Poisson model, such a factorization formula can be found in section 6.4.8 of Bühlmann (1970).

\section{THE DIVIDENDS-PENALTY IDENTITY}

We assume that at the time of ruin, a penalty is due. The penalty may depend on the deficit at ruin and possibly also on the surplus immediately before ruin. However, the penalty must not depend on the time of ruin. For the original surplus process, let $\phi(u)$ denote the expectation of the discounted penalty at ruin, considered a function of the initial surplus $u$. Such a function was introduced by Gerber and Shiu (1998). Similarly, $\phi(u ; b)$ denotes the expected discounted penalty at ruin, if dividends are paid according to the barrier strategy 
with barrier level $b$. The difference $\phi(u ; b)-\phi(u)$ can be expressed in terms of the difference at $u=b$ :

$$
\phi(u ; b)-\phi(u)=C(u, b)[\phi(b ; b)-\phi(b)], \quad u<b .
$$

This formula is obtained by "general reasoning": consider a particular sample path of the surplus process starting at $u<b$. Then the penalties at ruin (with and without the dividend barrier at $b$ ) can be different only, if the surplus reaches the level $b$ before ruin.

The (left) derivative of $\phi(u ; b)$ at $u=b$ is

$$
\phi^{\prime}(b-; b)=0 .
$$

This boundary condition is also best explained by heuristic reasoning. Consider situations 1 and 2 as in the justification of formula (5). In situation 2, it is quasi certain that the surplus arrives at the barrier $b$ before ruin. After that time, the sample paths (and hence the penalties at ruin) are identical.

In (7), we replace $C(u, b)$ by $h(u) / h(b)$ and take the derivative at $u=b$. Using (8) and (6), we obtain the formula

$$
\phi(u ; b)=\phi(u)-\phi^{\prime}(b) V(u ; b) .
$$

This dividends-penalty identity has the following interpretation: the change in penalty $\phi(u ; b)-\phi(u)$ is the expected present value of virtual dividends if the barrier strategy with parameter $b$ is applied, with the rate of these "dividends" being adjusted by the factor $-\phi^{\prime}(b)$.

For the shifted compound Poisson process, formula (9) was discovered by Lin et al. (2003), where it was derived via the general solution of an integrodifferential equation. Yuen et al. (2006) extended the result of Lin et al. to the case with interest.

Remark 3.1. Note that (7) is not limited to barrier strategies. It holds more generally for any strategy under which no dividends are paid whenever the surplus is below $b$. One such dividend strategy is the threshold strategy; then a constant fraction of the premium income is paid as dividends as long as the surplus is above the threshold $b$, see Gerber and Shiu (2006) and Lin and Pavlova (2006). However, the conditions (5) and (8) are only satisfied for the barrier strategy. Hence, the dividends penalty identity holds only for the barrier strategy.

\section{THE OPTIMAL DIVIDEND BARRIER}

How should the dividend barrier $b$ be chosen? A first idea, which goes back to De Finetti (1957), is to choose $b$ in order to maximize the expectation of the present value of all dividends until ruin. This is a natural idea, because the 
shareholders are the recipients of the dividends, and they decide upon the dividends policy. Thus the mathematical problem is to maximize $V(u ; b)$. In view of (6), an equivalent problem is to minimize $h^{\prime}(b)$. A set of references can be found in Gerber and Shiu (2004).

Dickson and Waters (2004) argued that the shareholders should be obliged to cover the deficit at ruin. As a consequence, they determine $b$ to maximize the difference between $V(u ; b)$ and the expected present value of the deficit at ruin. Gerber et al. (2006) further explore the impact of this assumption on the optimal dividend barrier.

We shall now consider the more general problem, where $b$ is chosen to maximize

$$
W(u ; b)=V(u ; b)-\phi(u ; b), \quad u \leq b,
$$

the difference between the expected present value of all dividends until ruin and the expected discounted value of the penalty at ruin. Here, the penalty at ruin is a given function of the deficit at ruin. It follows from (9) and (6) that

$$
W(u ; b)=h(u)\left[\frac{1+\phi^{\prime}(b)}{h^{\prime}(b)}\right]-\phi(u) .
$$

This shows that $b=b^{*}$ must be chosen in order to maximize the expression

$$
\frac{1+\phi^{\prime}(b)}{h^{\prime}(b)} \text {. }
$$

An important consequence of (11) is that the optimal value $b^{*}$ is independent of $u$ (as long as $u \leq b^{*}$ ). We note that the first order condition for $b^{*}$ is

$$
\phi^{\prime \prime}\left(b^{*}\right) h^{\prime}\left(b^{*}\right)-\left[1+\phi^{\prime}\left(b^{*}\right)\right] h^{\prime \prime}\left(b^{*}\right)=0 .
$$

From (5) and (8) it follows that

$$
W^{\prime}(b-; b)=1 .
$$

Furthermore, the left second derivative $W^{\prime \prime}(b-; b)$ vanishes, if $b=b^{*}$ :

$$
W^{\prime \prime}\left(b^{*}-; b^{*}\right)=0 \text {. }
$$

This can be seen as follows. From (11) we gather that

$$
W^{\prime \prime}(b-; b)=h^{\prime \prime}(b)\left[\frac{1+\phi^{\prime}(b)}{h^{\prime}(b)}\right]-\phi^{\prime \prime}(b) \text {. }
$$

Setting $b=b^{*}$ and using (13), we obtain (15). 
Remark 4.1. We limit our discussion to barrier strategies and we do not consider more general dividend strategies. However, we believe that the barrier strategy with parameter $b^{*}$ is optimal among all strategies, as long as $u \leq b^{*}$.

\section{INITIAL SURPLUS ABOVE THE BARRIER}

Up to now we have considered a barrier strategy with parameter $b$ and assumed that the initial surplus $u$ was at most $b$. In this section we consider the case where $u>b$. Then, there is an immediate dividend in the amount of $u-b$, which reduces the surplus to $b$. Hence, the expected present value of all dividends until ruin is now

$$
V(u ; b)=u-b+V(b ; b), \quad u>b .
$$

The objective function is obtained by subtraction of $\phi(b ; b)$. It is

$$
W(u ; b)=u-b+W(b ; b), \quad u>b .
$$

Thus, $W^{\prime}(u ; b)=1$ and $W^{\prime \prime}(u ; b)=0$ for $u>b$. From (14) it follows that $W^{\prime}(u ; b)$ is a continuous function of $u$ at $u=b$, and from (15) it follows that $W^{\prime \prime}(u ; b)$ is a continuous function of $u$ at $u=b^{*}$. The latter condition is generally known as the smooth pasting or high contact condition (for optimality).

Let us now consider the problem of maximizing $W(u ; b)$ if $u>b$. Thus the problem is to choose $b$ in order to maximize

$$
-b+W(b ; b)=-b+h(b)\left[\frac{1+\phi^{\prime}(b)}{h^{\prime}(b)}\right]-\phi(b)
$$

by (11) with $u=b$. The derivative of this function of $b$ is

$$
h(b)\left[\frac{1+\phi^{\prime}(b)}{h^{\prime}(b)}\right]^{\prime} .
$$

We note that the sign of this expression is the same as the sign of the derivative of (12). This shows that the local maxima of (11) and (18) are attained for the same values of $b$. In particular, (18) has at least a local maximum at $b=b^{*}$. We expect that in many cases (18) attains its global maximum at $b=b^{*}$; then $W(u ; b)$ is maximized by $b=b^{*}$, even if $u>b^{*}$. However, we do not know if this is true in general.

In the following two sections, we shall consider two classical models of risk theory, where the calculations can be done in a transparent fashion. This should not distract from the fact that the underlying theory is quite general. 


\section{THE WIENER PROCESS}

As a first illustration, assume that the surplus before dividends is a Wiener process with expected gains per unit time $\mu>0$ and variance per unit time $\sigma^{2}$. In this model, the surplus at ruin is 0 . Hence, the penalty at ruin is a constant $\Pi>0$. Here $h(u)$ and $\phi(u)$ satisfy the same differential equation. For example,

$$
\frac{\sigma^{2}}{2} h^{\prime \prime}(u)+\mu h^{\prime}(u)-\delta h(u)=0 ;
$$

see for example Gerber and Shiu (2004). If the initial surplus is zero, ruin is immediate. Hence $h(0)=0$ and $\phi(0)=\Pi$. If the initial surplus is large, ruin occurs late or not at all. Hence $\phi(\infty)=0$ because of the discounting. From these boundary conditions it follows that

$$
\begin{aligned}
& h(u)=e^{r u}-e^{s u} \text { (apart from a constant factor), } \\
& \phi(u)=\Pi e^{s u}
\end{aligned}
$$

where $r>0, s<0$ are the solutions of the characteristic equation

$$
\frac{\sigma^{2}}{2} \xi^{2}+\mu \xi-\delta=0
$$

From (11) and (22) we obtain

$$
W(u ; b)=\left(e^{r u}-e^{s u}\right)\left[\frac{1+s \Pi e^{s b}}{r e^{r b}-s e^{s b}}\right]-\Pi e^{s u} .
$$

Now $W(u ; b)$ satisfies also the differential equation

$$
\frac{\sigma^{2}}{2} W^{\prime \prime}(u ; b)+\mu W^{\prime}(u ; b)-\delta W(u ; b)=0, \quad 0 \leq u \leq b .
$$

We use this equation for $u=b$ and then set $b=b^{*}$. By using (14) and (15), we see that

$$
W\left(b^{*} ; b^{*}\right)=\frac{\mu}{\delta},
$$

the present value of a perpetuity, independent of $\Pi$. It follows that $b^{*}$ is an increasing function of $\Pi$.

We can use (26) to determine the optimal dividend barrier; because of (24), $b=b^{*}$ is the solution of

$$
\frac{e^{r b}-e^{s b}}{r e^{r b}-s e^{s b}}-\Pi \frac{(r-s) e^{(r+s) b}}{r e^{r b}-s e^{s b}}=\frac{\mu}{\delta} .
$$


TABLE 1

THE INFLUENCE OF $\mu$ AND $\Pi$ ON $b^{*}$ AND the VALUeS OF $\Pi^{c}$

\begin{tabular}{c|c|c|c|c|c|c|c|c|c}
\hline \hline$\mu$ & $\Pi=0$ & $\Pi=1$ & $\Pi=2$ & $\Pi=3$ & $\Pi=4$ & $\Pi=5$ & $\Pi=10$ & $\Pi=100$ & $\Pi^{c}$ \\
\hline 0.5 & 9.02 & 9.74 & $(10.43)$ & $(11.08)$ & $(11.70)$ & $(12.29)$ & $(14.85)$ & $(30.36)$ & 1.37 \\
1.0 & 14.15 & 14.53 & 14.89 & 15.23 & 15.56 & 15.87 & 17.25 & $(26.80)$ & 23.65 \\
1.5 & 15.98 & 16.18 & 16.37 & 16.56 & 16.73 & 16.90 & 17.68 & 23.82 & 462.39 \\
2.0 & 16.20 & 16.31 & 16.42 & 16.53 & 16.63 & 16.74 & 17.20 & 21.39 & 27349.70 \\
2.5 & 15.75 & 15.83 & 15.90 & 15.96 & 16.03 & 16.10 & 16.40 & 19.41 & $6.45 \times 10^{6}$ \\
3.0 & 15.08 & 15.12 & 15.17 & 15.22 & 15.26 & 15.31 & 15.52 & 17.78 & $6.18 \times 10^{9}$ \\
3.5 & 14.34 & 14.38 & 14.41 & 14.44 & 14.48 & 14.51 & 14.67 & 16.42 & $2.37 \times 10^{13}$ \\
4.0 & 13.62 & 13.65 & 13.67 & 13.70 & 13.72 & 13.75 & 13.87 & 15.27 & $3.62 \times 10^{17}$ \\
4.5 & 12.95 & 12.97 & 12.99 & 13.01 & 13.03 & 13.05 & 13.14 & 14.28 & $2.17 \times 10^{22}$ \\
5.0 & 12.32 & 12.34 & 12.36 & 12.37 & 12.39 & 12.40 & 12.48 & 13.43 & $5.10 \times 10^{27}$ \\
\hline \hline
\end{tabular}

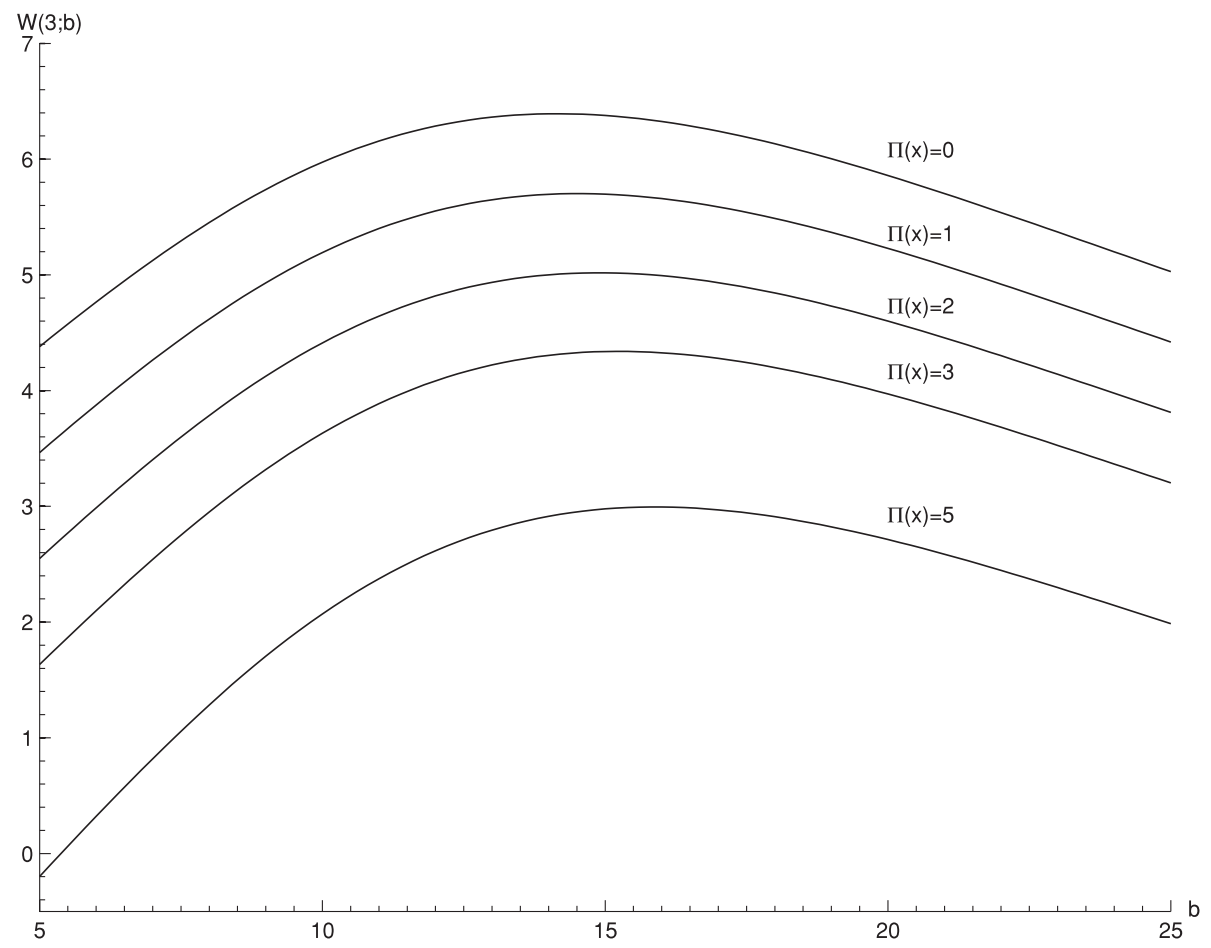

FIGURE $1 . W(3 ; b)$ as a function of $b$ (Brownian motion model).

If $\Pi=0$, (27) can be solved explicitly. We find that

$$
b^{*}=\frac{1}{r-s} \ln \frac{1-s \frac{\mu}{\delta}}{1-r \frac{\mu}{\delta}}=\frac{2}{r-s} \ln \left(-\frac{s}{r}\right),
$$


because $\frac{\mu}{\delta}=\frac{r+s}{r s}$. We note that (26) for $\Pi=0$ and (28) can be found in Gerber and Shiu (2004).

A company with surplus $u$ has an economic interest to pursue its activity, only if $u<W\left(u ; b^{*}\right)$. This condition is violated if the penalty $\Pi$ is too large. Thanks to (26) we obtain a closed form expression for the critical value $\Pi^{c}$ of the penalty. Setting $b=\frac{\mu}{\delta}$ in (27) and solving for $\Pi$, we obtain

$$
\Pi^{c}=\frac{1}{r-s}\left[\left(1-\frac{\mu}{\delta} r\right) e^{-s \frac{\mu}{\delta}}-\left(1-\frac{\mu}{\delta} s\right) e^{-r \frac{\mu}{\delta}}\right] .
$$

Thus for $\Pi \geq \Pi^{c}$, the company has no economic interest in the business.

Example 6.1. Assume $\delta=0.05$ and $\sigma^{2}=15$. Table 1 shows $b^{*}$ as $\mu$ and $\Pi$ vary. The numbers confirm that $b^{*}$ is an increasing function of $\Pi$. This can also be seen in Figure 1, which, for $\mu=1$, displays the graph of $W(3 ; b)$ for different values of $\Pi$. However, $b^{*}$ is not a monotone function of $\mu$. The critical values of $\Pi$ are shown in the last column of Table 1 . They play a role for small values of $\mu$ only. If $b^{*}>W\left(b^{*} ; b^{*}\right)$, the value of $b^{*}$ is in parentheses.

\section{THE SHIFTED COMPOUND POISSON PROCESS}

In this section we consider the classical model, where the surplus of a company is of the form (1). The functions $h(u)$ and $\phi(u)$ are solutions of the integrodifferential equations

$$
c h^{\prime}(u)-(\lambda+\delta) h(u)+\lambda \int_{0}^{u} h(x) p(u-x) d x=0
$$

and

$$
c \phi^{\prime}(u)-(\lambda+\delta) \phi(u)+\lambda \int_{0}^{u} \phi(x) p(u-x) d x+\lambda \int_{0}^{u} \Pi(y) p(u+y) d y=0 .
$$

Here $p(x)$ is the claim amount probability density function, and $\Pi(x)$ is the penalty, if the deficit at ruin is $x$.

We consider the case where $p(x)$ is a mixture of exponential probability density functions,

$$
p(x)=\sum_{i=1}^{n} A_{i} \beta_{i} e^{-\beta_{i} x},
$$

with $0<\beta_{1}<\beta_{2}<\cdots<\beta_{n}, A_{i}>0$, and $A_{1}+\cdots+A_{n}=1$. Then (31) can be written as

$$
c \phi^{\prime}(u)-(\lambda+\delta) \phi(u)+\lambda \int_{0}^{u} \phi(x) p(u-x) d x+\lambda \sum_{i=1}^{n} A_{i} e^{-\beta_{i} u} \Pi_{i}=0,
$$


with

$$
\Pi_{i}=\beta_{i} \int_{0}^{\infty} \Pi(y) e^{-\beta_{i} y} d y
$$

Note that $\Pi_{i}$ is the conditional expectation of the penalty at ruin, given that the claim that leads to ruin is of type $i$.

We apply the operator

$$
\prod_{i=1}^{n}\left(\frac{d}{d u}+\beta_{i}\right)
$$

to (30) and (33). Because

$$
\left(\frac{d}{d u}+\beta\right) e^{-\beta u}=0
$$

we obtain in both cases a linear homogenous differential equation of order $n+1$ with constant coefficients, which are the same for both. Hence we try

$$
\begin{aligned}
& h(u)=\sum_{k=0}^{n} C_{k} e^{\rho_{k} u}, \\
& \phi(u)=\sum_{k=0}^{n} D_{k} e^{\rho_{k} u} .
\end{aligned}
$$

We substitute (37) and (32) in (30). Comparing the coefficients of $e^{\rho_{k} u}$ in the resulting equation, we see that $\rho_{0}, \rho_{1}, \cdots, \rho_{n}$ must be the solution of Lundberg's equation,

$$
c \xi-(\lambda+\delta)+\lambda \sum_{i=1}^{n} \frac{A_{i} \beta_{i}}{\beta_{i}+\xi}=0 .
$$

This equation has exactly $n+1$ solutions and

$$
-\beta_{n}<\rho_{n}<-\beta_{n-1}<\cdots<-\beta_{1}<\rho_{1}<0<\rho_{0} .
$$

Then comparing the coefficients of $e^{-\beta_{i} u}$, we see that

$$
\sum_{k=0}^{n} C_{k} \frac{1}{\beta_{i}+\rho_{k}}=0, \quad i=1, \cdots, n .
$$

These are $n$ equations for $C_{0}, C_{1}, \cdots, C_{n}$, which are determined only up to a common factor. Formula (D.31) of Chan et al. (2006) contains a closed form expression for the $C_{k}$ 's. 
Now we turn to the discounted penalty function. From $\phi(\infty)=0$ and $\rho_{0}>0$, it follows that $D_{0}=0$. We substitute (38) and (32) in (33). Comparing the coefficients of $e^{-\beta_{i} u}$, we see that

$$
\sum_{k=1}^{n} D_{k} \frac{1}{\beta_{i}+\rho_{k}}=\frac{\prod_{k}}{\beta_{i}}, \quad i=1, \cdots, n .
$$

Then $D_{1}, \cdots, D_{n}$ must be determined from this system of linear equations. As pointed out by Gerber and Shiu (2006, Appendix A), the coefficient matrix of this system is a Cauchy matrix, and there is a closed form expression for the inverse of such a matrix. A closed form expression for the solutions of (42) is contained in formula (7.9) of Gerber and Shiu (2005).

Finally, $b=b^{*}$ is determined in order to maximize the expression (12), which is

$$
\frac{1+\sum_{k=1}^{n} D_{k} \rho_{k} e^{\rho_{k} b}}{\sum_{k=0}^{n} C_{k} \rho_{k} e^{\rho_{k} b}} .
$$

For an illustration, we consider the following mixture of exponential probability density functions:

$$
p(x)=\frac{1}{3}\left(\frac{1}{2} e^{-x / 2}\right)+\frac{2}{3}\left(2 e^{-2 x}\right), \quad x>0 .
$$

It has mean 1 and second moment 3 . Hence by setting $\lambda=5$, the compound Poisson process $\{S(t)\}$ is comparable to the Wiener process in Example 6.1. In the following example, the calculations are done for $c=5.5,6,6.5 ., \cdots, 10$, which corresponds to $\mu=0.5,1,1.5, \cdots, 5$. As in Example 6.1, $\delta=0.05$.

Example 7.1. We consider three families of elementary penalty functions, the constant penalty function $\Pi(x)=\Pi$ (thus $\Pi_{i}=\Pi$ in (34)), the function $\Pi(x)=$ $K x\left(\Pi_{i}=K / \beta_{i}\right)$ where the penalty is proportional to the deficit at ruin, and the function $\Pi(x)=x+K x^{2}\left(\Pi_{i}=1 / \beta_{i}+K / \beta_{i}^{2}\right)$ where the penalty is the sum of the deficit at ruin and a term that is proportional to the square of the deficit at ruin. The optimal dividend barriers are displayed in Tables 2-4. Whenever $b^{*}>$ $W\left(b^{*} ; b^{*}\right)$, the value of $b^{*}$ is shown in parentheses. It is interesting to compare Table 2 with Table 1 . For small security loadings (upper part of the tables), the optimal dividend barrier in the Wiener process model is higher. In each column there is a crossover. In the lower part of the tables, the optimal dividend barrier in the compound Poisson process model is substantially higher. In Table 3, $b^{*}$ is an increasing function of $K$. But we observe that $b^{*}$ increases very slowly with $K$. The values for $K=0$ and $K=1$ can be found in Tables 3 and 4 of Gerber et al. (2006). For $c=6$ and $K=1$, we have $b^{*}=12$.98. Figure 3 shows that $W(u ; b)$ is maximized by $b=b^{*}$ not only if $u<b^{*}$, but also, if $u>b^{*}$. Table 4 has a similar pattern as Table 3 . In all three tables we observe that $b^{*}$ increases 
TABLE 2

THE INFLUENCE OF $c$ AND $\Pi$ ON $b^{*}$ WHEN $\Pi(x)=\Pi$

\begin{tabular}{c|r|r|r|r|r|r|r|c}
\hline \hline \multicolumn{1}{c|}{$c$} & $\Pi=0$ & $\Pi=1$ & $\Pi=2$ & $\Pi=3$ & $\Pi=4$ & $\Pi=5$ & $\Pi=10$ & $\Pi=100$ \\
\hline 5.5 & 5.72 & 6.50 & 7.27 & 8.01 & $(8.71)$ & $(9.39)$ & $(12.35)$ & $(30.51)$ \\
6.0 & 12.10 & 12.58 & 13.05 & 13.49 & 13.91 & 14.32 & 16.11 & $(28.44)$ \\
6.5 & 15.57 & 15.86 & 16.14 & 16.40 & 16.66 & 16.91 & 18.03 & 26.82 \\
7.0 & 17.36 & 17.54 & 17.72 & 17.89 & 18.06 & 18.23 & 18.98 & 25.57 \\
7.5 & 18.29 & 18.41 & 18.54 & 18.66 & 18.78 & 18.89 & 19.43 & 24.60 \\
8.0 & 18.78 & 18.87 & 18.96 & 19.05 & 19.14 & 19.23 & 19.64 & 23.84 \\
8.5 & 19.04 & 19.12 & 19.19 & 19.26 & 19.33 & 19.39 & 19.72 & 23.23 \\
9.0 & 19.19 & 19.24 & 19.30 & 19.36 & 19.41 & 19.47 & 19.73 & 22.74 \\
9.5 & 19.26 & 19.31 & 19.36 & 19.40 & 19.45 & 19.49 & 19.71 & 22.33 \\
10.0 & 19.30 & 19.34 & 19.38 & 19.42 & 19.46 & 19.49 & 19.68 & 21.99 \\
\hline \hline
\end{tabular}

TABLE 3

THE INFLUENCE OF $c$ AND $K$ ON $b^{*}$ WHEN $\Pi(x)=K x$

\begin{tabular}{r|r|r|c|c|c|c|c|c}
\hline \hline \multicolumn{1}{c|}{$c$} & $K=0$ & $K=1$ & $K=2$ & $K=3$ & $K=4$ & $K=5$ & $K=10$ & $K=100$ \\
\hline 5.5 & 5.72 & 7.15 & $(8.50)$ & $(9.74)$ & $(10.89)$ & $(11.94)$ & $(16.17)$ & $(36.26)$ \\
6.0 & 12.10 & 12.98 & 13.79 & 14.54 & 15.23 & 15.88 & $(18.52)$ & $(32.77)$ \\
6.5 & 15.57 & 16.10 & 16.59 & 17.06 & 17.49 & 17.90 & 19.63 & $(30.24)$ \\
7.0 & 17.36 & 17.70 & 18.02 & 18.33 & 18.62 & 18.89 & 20.10 & 28.39 \\
7.5 & 18.29 & 18.52 & 18.75 & 18.97 & 19.17 & 19.37 & 20.27 & 26.99 \\
8.0 & 18.78 & 18.95 & 19.12 & 19.29 & 19.44 & 19.59 & 20.29 & 25.92 \\
8.5 & 19.04 & 19.18 & 19.31 & 19.44 & 19.56 & 19.68 & 20.24 & 25.07 \\
9.0 & 19.19 & 19.30 & 19.40 & 19.51 & 19.61 & 19.71 & 20.16 & 24.39 \\
9.5 & 19.26 & 19.35 & 19.44 & 19.53 & 19.61 & 19.69 & 20.08 & 23.84 \\
10.0 & 19.30 & 19.37 & 19.45 & 19.52 & 19.59 & 19.67 & 20.00 & 23.38 \\
\hline \hline
\end{tabular}

TABLE 4

THE INFLUENCE OF $c$ AND $K$ ON $b^{*}$ WHEN $\Pi(x)=x+K x^{2}$

\begin{tabular}{c|r|r|c|c|c|c|c|c}
\hline \hline$c$ & $K=0$ & $K=0.1$ & $K=0.2$ & $K=0.3$ & $K=0.4$ & $K=0.5$ & $K=1$ & $K=10$ \\
\hline 5.5 & 7.15 & 7.69 & $(8.22)$ & $(8.73)$ & $(9.22)$ & $(9.70)$ & $(11.86)$ & $(27.77)$ \\
6.0 & 12.98 & 13.31 & 13.62 & 13.93 & 14.23 & 14.51 & 15.83 & $(26.49)$ \\
6.5 & 16.10 & 16.30 & 16.49 & 16.68 & 16.86 & 17.04 & 17.87 & 25.37 \\
7.0 & 17.70 & 17.83 & 17.95 & 18.08 & 18.20 & 18.32 & 18.88 & 24.44 \\
7.5 & 18.52 & 18.61 & 18.70 & 18.79 & 18.88 & 18.96 & 19.36 & 23.68 \\
8.0 & 18.95 & 19.02 & 19.09 & 19.15 & 19.22 & 19.28 & 19.59 & 23.07 \\
8.5 & 19.18 & 19.23 & 19.28 & 19.34 & 19.39 & 19.44 & 19.68 & 22.58 \\
9.0 & 19.30 & 19.34 & 19.38 & 19.42 & 19.46 & 19.50 & 19.70 & 22.17 \\
9.5 & 19.35 & 19.39 & 19.42 & 19.46 & 19.49 & 19.52 & 19.69 & 21.83 \\
10.0 & 19.37 & 19.40 & 19.43 & 19.46 & 19.49 & 19.52 & 19.66 & 21.55 \\
\hline \hline
\end{tabular}




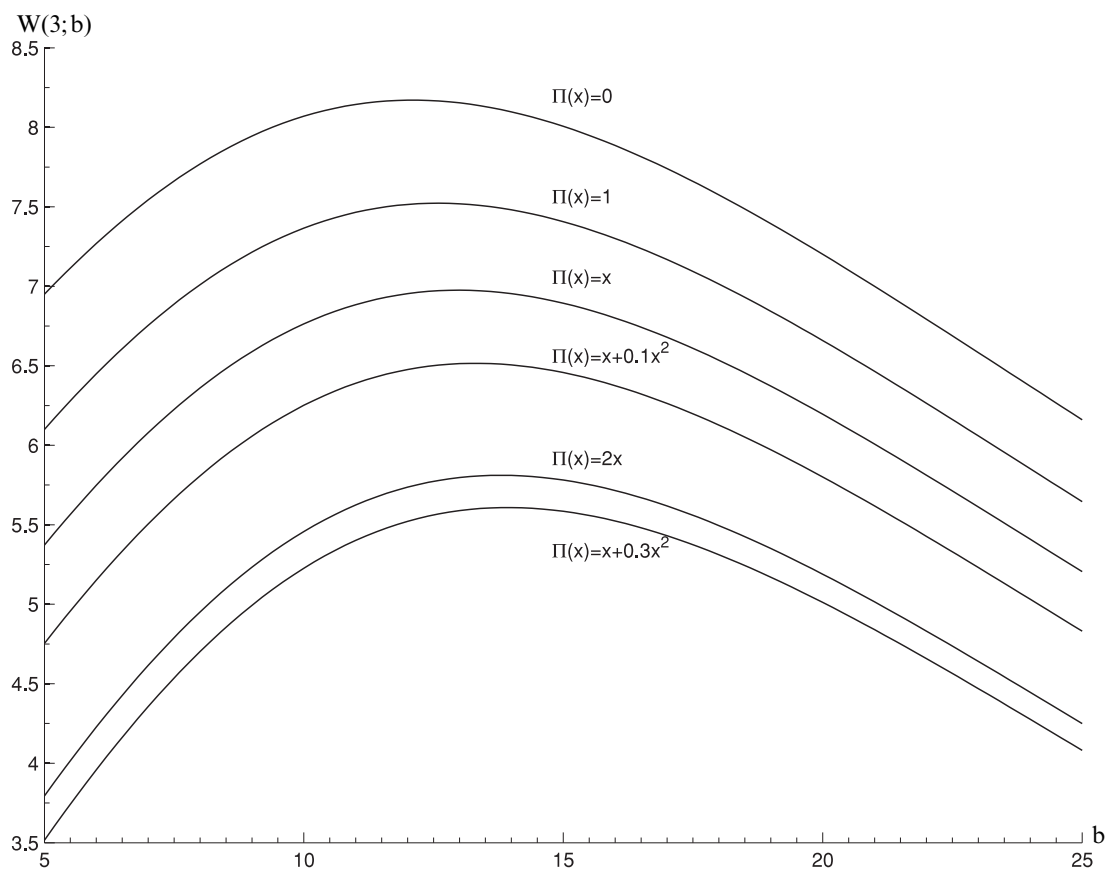

Figure 2. $W(3 ; b)$ as a function of $b$ (Compound Poisson model).

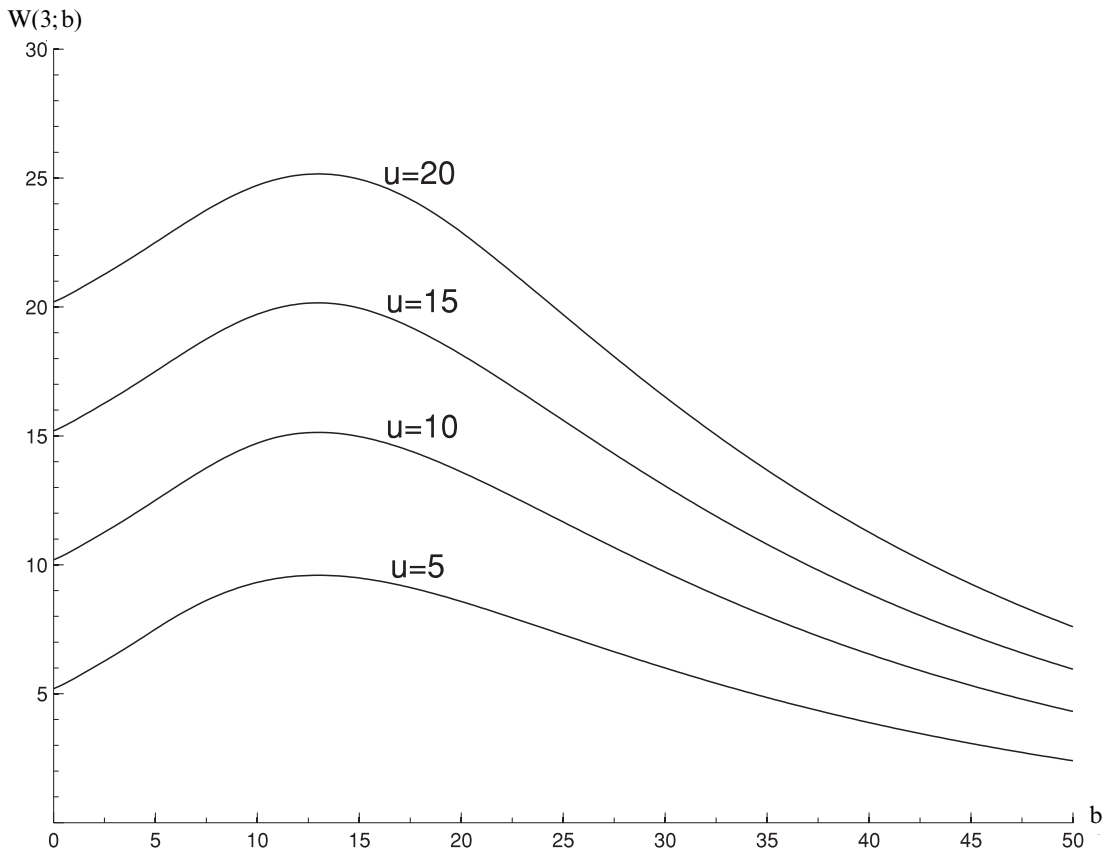

Figure 3. $W(u ; b)$ as a function of $b$ when $\Pi(x)=x$ and $c=6$. 
with $c$ up to a certain point and decreases thereafter. Figure 2 displays the graphs of $W(3 ; b)$ when $c=6$ for different penalty functions.

\section{ACKNOWLEDGMENTS}

The authors are grateful to Elias S.W. Shiu for his advice and to two anonymous referees for their comments. X.S. Lin would like to acknowledge the Natural Sciences and Engineering Research Council of Canada, and that part of the work was completed when he was visiting the Department of Applied Mathematics, Hong Kong Polytechnic University. H. Yang would like to acknowledge the Research Grants Council of the Hong Kong Special Administrative Region, China (Project No. HKU 7239/04H).

\section{REFERENCES}

Albrecher, H., Claramunt, M.M. and Mármol, M. (2005) "On the distribution of dividend payments in a Sparre Andersen model with generalized Erlang(n) interclaim times", Insurance: Mathematics and Economics, 37, 324-334.

Bühlmann, H. (1970) Mathematical Methods in Risk Theory, Springer-Verlag, Berlin.

Chan, B., Gerber, H.U. and Shiu, E.S.W. (2006) "Discussion of Xiaowen Zhou's 'On a classical risk model with a constant dividend barrier"”, North American Actuarial Journal, 10(2), 133-139.

De FinetTi, B. (1957) "Su un' impostazione alternativa della teoria collettiva del rischio," Transactions of the XVth International Congress of Actuaries, 2, 433-443.

Frostig, E. (2005) "The expected time to ruin in a risk process with constant barrier via martingales", Insurance: Mathematics and Economics, 37, 216-228.

DiCKSON, D.C.M. and WATERS, H.R. (2004) "Some optimal dividends problems", ASTIN Bulletin, 34, 49-74.

Gerber, H.U. (1972) "Games of economic survival with discrete- and continuous-income process", Operations Research, 20(1), 37-45.

Gerber, H.U. and Shiu, E.S.W. (1998) "On the time value of ruin", North American Actuarial Journal, 2(1), 48-78.

Gerber, H.U. and SHIU, E.S.W. (2004) "Optimal dividends: analysis with Brownian motion," North American Actuarial Journal 8(1), 1-20.

Gerber, H.U. and Shiu, E.S.W. (2005) "The time value of ruin in a Sparre Andersen model," North American Actuarial Journal 9(2), 49-84.

Gerber, H.U. and ShiU, E.S.W. (2006) "On optimal dividend strategies in the compound Poisson model”, North American Actuarial Journal 10(2), 76-93.

Gerber, H.U., Shiu, E.S.W. and Smith, N. (2006) "Maximizing dividends without bankruptcy", ASTIN Bulletin 36, 5-23.

Li, S. and GARrido, J. (2004) "On a class of renewal models with a constant dividend barrier", Insurance: Mathematics and Economics, 35, 697-701.

Lin, X.S. and Pavlova, K. (2006) "The compound Poisson risk model with a threshold dividend strategy", Insurance: Mathematics and Economics, 38, 57-80.

Lin, X.S., Willmot, G.E. and Drekic, S. (2003) "The classical risk model with a constant dividend barrier: analysis of the Gerber-Shiu discounted penalty function", Insurance: Mathematics and Economics, 33, 551-566.

YUEN, K.C., WANG, G. and LI, W.K. (2006) "The Gerber-Shiu expected discounted penalty function for risk processes with interest and a constant dividend barrier", Insurance: Mathematics and Economics, To appear. 
HANS U. GERBER

Distinguished Visiting Professor at the University of Hong Kong

École des hautes études commerciales

Université de Lausanne, CH-1015 Lausanne

Switzerland

E-mail:hgerber@unil.ch.

X. SHELDON LIN

Department of Statistics

University of Toronto

Toronto, Ontario M5S $3 G 3$ Canada

E-mail: sheldon@utstat.utoronto.ca.

HAILIANG YANG

Department of Statistics and Actuarial Science

University of Hong Kong

Pokfulam Road, Hong Kong

E-mail:hlyang@hkusua.hku.hk. 